${ }^{1} \mathrm{~F}$ Abbasi, ${ }^{2} \mathrm{P}$ Vahdani, ${ }^{3} \mathrm{~S}$ Korooni, ${ }^{2} \mathrm{~A}$ Naderi. 'Bushehr University of Medical Sciences, Bushehr; ${ }^{2}$ Infectious Diseases and Tropical Medicine Research Center, Shaheed Beheshti Medical University, Tehran; ${ }^{3}$ Shiraz University of Medical Sciences, Shiraz, Iran

Background and Aims Hydatidosis, caused by Echinococcus granulosus, is an endemic parasitic disease. The most frequent anatomic locations are liver and lung. Dogs and other canines are the primary definitive hosts for this parasite. Hydatid cyst may develop after accidental ingestion of tapeworm eggs, excreted with the feces of these animals. Diagnosis is usually based on radiological and serological findings.

Patient We present a 6 Year-Old-Boy with abdominal pain since months ago. He had history of travelling to village and exposure to cattle and dog. Physical examination of head and neck, chest, extremities, and neurologic exam were normal. In abdominal Physical examination he had mild right upper quadrant tenderness without rebound tenderness. Liver was palpable $3 \mathrm{Cm}$ below costal margin. Abdomoinal sonography and CT scan showed multiple huge cysts in liver that occupied almost all the liver space. Serum hydatid cyst antibody was positive. With diagnosis of hydatosis surgery was done and albendazole started for him. The patient improved after a while and he was discharged with good general condition.

Conclusion Hydatid cyst should be considered in every patient with liver cystic lesion in endemic area.

\section{FATAL HUMAN BOCAVIRUS INFECTION IN A BOY WITH IPEX-LIKE SYNDROME AND VACCINE-ACQUIRED ROTAVIRUS ENTERITIS AWAITING STEM CELL TRANSPLANTATION}

doi:10.1136/archdischild-2012-302724.0958

'D Klinkenberg, 'R Kobbe, ${ }^{2} \mathrm{R}$ Schneppenheim, ${ }^{2} \mathrm{M}$ Müller, 'M Blohm, ${ }^{3} \mathrm{M}$ Malecki, ${ }^{3} \mathrm{~V}$ Schildgen, ${ }^{3} 0$ Schildgen. ${ }^{1}$ Clinic of Paediatrics; ${ }^{2}$ Clinic for Paediatric Haematology

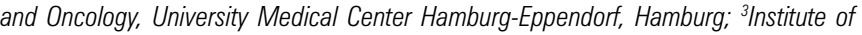
Pathology, Kliniken der Stadt Köln, Köln, Germany

We report about a 21-month-old boy presenting with chronic diarrhea and obstructive lung disease since infancy. His older brother, the first male child of non-consanguine Egyptean parents, had died at the age of 20 months suffering from BCGitis and severe CMV infection, suggesting a severe primary immundeficiency syndrome.

We found immunological dysregulation, endocrine dysfunction and enteropathy compatible with IPEX syndrome, therefore the child was listed for stem cell transplantation (SCT). FOXP3 gene showed no IPEX associated mutations, but sequencing IL2RG we found an mutation in Exon 2 associated with x-linked IPEX-like SCID (c.252C>A, p.N84K).

The pulmonary condition of the boy deteriorated and he was admitted on the ICU, where he was mechanically ventilated ever since. After common respiratory infections were excluded, chronic human bocavirus (hBoV) was identified by multiplex-PCR as the primary causative pulmonary agent causing his respiratory failure. $\mathrm{HBoV}$ was found in decreasing quantity in respiratory material, blood and stool specimen.

Interestingly, chonic rotavirus shedding was notified in repetitive stool specimen. As the boy had been vaccinated against rotavirus, vaccine-aquired chronic infection was suspected and confirmed by typing of a vaccine virus specific gene variant.

The boy died before SCT could be preformed.

Since its dicovery, there is an ongoing discussion if $\mathrm{hBoV}$ can cause serious infections or represents only a harmless "bystander". This case report shows that $\mathrm{hBoV}$ can result in lethal respiratory infections in immunocompromised children. JOHNSON SYNDROME IN TWO CHILDREN

doi:10.1136/archdischild-2012-302724.0959
L Sfaihi, K Baklouti, I Maaloul, A Hakim, H Aloulou, T Kamoun, M Hachicha. CHU Hedi Chaker, Sfax, Tunisia

Background Mycoplasma pneumonia (MP) infections are often asymptomatic but can involve multiple organ systems. Secondary skin reactions are common, although few patients infected develop Stevens Johnson syndrome (SJS).

Results We describe 2 cases of Mycoplasma pneumoniae chest infection associated with Stevens Johnson syndrome. The two patients had prodromal symptoms of an upper respiratory tract infection before the onset of the eruption. The patients had extensive epidermal bullous vesicles, oropharyngeal and genital ulceration, injected conjunctivae and sclera and swollen lips with flaccid bullae. The mycoplasma IgG and IgM titers returned positive, and blood cultures and other titers were negative. They were successfully treated with macrolides and glucocorticoids

Conclusion Although the clinical course may be severe and prolonged, the prognosis is uniformly good with complete recovery.

\section{SECONDARY PSEUDOHYPOALDOSTERONISM DUE TO PYELONEPHRITIS: TWO CASES REPORT}

doi:10.1136/archdischild-2012-302724.0960

II Llana, ${ }^{2} \mathrm{MD}$ Sanchez-Redondo, 'M Benedit, ${ }^{2} \mathrm{~J}$ Cobas, 'MT Garcia de Alvaro. 'Hospital HM Torrelodones, Madrid; ${ }^{2}$ Hospital Virgen de la Salud, Toledo, Spain

Secondary pseudohypoaldosteronism occurs due to transient resistance in renal tubules causing renal sodium loss, hyponatremia and hyperkaliemia. This may resemble congenital adrenal hyperplasia. This is to be considered specially when a urinary tract infection is present as treatment and prognosis varies.

We report two infants who developed pseudohypoaldosteronism secondary to acute tract urinary infection and urologic malformations.

Patient 127 days old infant, weight: 4056 gr. He suffers severe dehydration, hyponatremia $119 \mathrm{mmol} / \mathrm{L}, \mathrm{K} 7 \mathrm{mmol} / \mathrm{L}$ and metabolic acydosis, normal serum creatinine and normal $17 \mathrm{OH}$ progest. Pathologycal urine analysis and hight leves of serum rennin and aldosterone. Urine culture: E.Coli. We can also apreciate left Ultrasonography hidronefrosis IV/V and primary megaureter. He was treated with Ampiciline + gentamicine and ions stabilisation within the following 36 hours after hospital admission.

Patient 214 days old infant, 2510 gr. Admitted at emergencies with a severe deshydration. Hyponatremia and hyperpotasemia that needed urgent treatment and severe metabolic acydosis. Electrolitic disorders were amended, then a urine culture was done with positive results in E. Coli test. He was treated with ampiciline + gentamicine. The renal scan evinced a bilateral ureteral hydronephrosis. The infant showed a recovery thanks to antibiotics treatment.

Conclusions An endocrinological evaluation is necessary to a diagnosis but we musn't forget the urinary cultures.

Most probable cause is the inmature renal responsiveness to aldosterone in three first months of life when exist urinary tract anomalies and/or urinary tract infection.

This secondary pseudohypoaldosteronism is reversible with early treatment.

\section{THE DIAGNOSTIC VALUE OF NEUROIMAGINIG IN RECOGNIZING AND THERAPY MONITORING OF ENCEPHALITIS AND MENINGITIS IN CHILDREN}

doi:10.1136/archdischild-2012-302724.0961

1J Surmik, '2E Emich-Widera, 'J Wróblewska, "I Maruniak-Chudek, ${ }^{3} E$ Kluczewska. ${ }^{1}$ Department of Neonatal Intensive and Special Care; ${ }^{2}$ Department of Neuropediatrics, 